Artigo Original

Hegemonia - Revista Eletrônica do Programa de Mestrado em Direitos Humanos, Cidadania e

Violência/Ciência Política do Centro Universitário Unieuro

ISSN: 1809-1261

UNIEURO, Brasília, número 27 (Especial), 2019, pp. 188-199.

Recebido em: 7/9/2018

Avaliado em: 5/10/2018

Aprovado em: 8/11/2018

\title{
DOENÇA DE ALZHEIMER: UM OLHAR FRENTE AO CUIDADOR FAMILIAR
}

Juliana Cavalcante de Arêdo, ${ }^{1}$ Valeria Alves Ferreira Soares da Rocha ${ }^{2}$ e Iolanda Bezerra dos

Santos Brandão ${ }^{3}$

Resumo: A população idosa vem crescendo no mundo e no Brasil, com seu avanço populacional e o aumento da expectativa de vida, surgem diferentes tipos de doenças, sendo a Doença de Alzheimer (DA), umas das demências mais neurodegenerativa progressiva e fatal. O sintoma inicial da doença é caracterizado pela perda progressiva da memória recente. $\mathrm{Na}$ evolução da mesma, outras funções também serão identificadas com o comprometimento cognitivo, deambulação prejudicada, linguagem alterada até incontinência urinária e fecal. Objetivo:Discutir o impacto da doença de Alzheimer (DA) frente à família e/ ou cuidador. Método:Trata-se de revisão da Literatura onde foram selecionados artigos organizados em bases de dados como Scielo, Lilacs, revistas Periódicas, Teses, Livro de tratado de Geriatria e Gerontologia, Protocolos e Diretrizes do Ministério da Saúde, site virtual da Associação Brasileira do Alzheimer. Os dados foram coletados entre anos de 2010 a 2017. Conclusão: O cuidador familiar e todos os familiares sofrem muitos impactos emocionais e físicos com a convivência com o portador, no entanto alguns grupos de apoio são disponibilizados, para sanar algumas dúvidas, questionamento e saber lidar com este portador na evolução da doença.

Palavras-chave: Doença de Alzheimer; Cuidador; Cuidador familiar; impacto emocional e Físico; enfrentamento diante do diagnóstico.

Abstract: The elderly population is increasing around the world and in Brazil, with its population expansion there are an increase in life expectancy. Different types of diseases arise, being Alzheimer's Disease (AD) one of the most progressive and fatal neurodegenerative dementias. The initial symptom of the disease is characterized by progressive loss of recent memory. In the evolution of the same, other functions will also be identified with cognitive impairment, impaired ambulation, altered language until urinary and fecal incontinence. Objective: To discuss the impact of Alzheimer's disease (AD) on the family and / or caregiver. Method: This is a review of the Literature in which articles organized in databases such as Scielo, Lilacs, Periodicals, Theses, Geriatrics and Gerontology Treaties, Protocols and Guidelines of the Ministry of Health, virtual website of the Brazilian Association of Alzheimer's disease. The data were collected between the years of 2010 and 2017. Conclusion: The family caregiver and all the family members suffer many emotional and physical impacts with the coexistence with the bearer, however some support

\footnotetext{
${ }^{1}$ Enfermeira. Especialista em Saúde do Idoso Gestão em Assistência em Gerontologia da Faculdade Laboro.

${ }^{2}$ Fonoaudióloga. Especialista em Saúde do Idoso Gestão em Assistência em Gerontologia da Faculdade Laboro.

3 Professora e Pesquisadora do Centro Universitário UNIEURO e Faculdade Laboro.
} 
Artigo Original

Hegemonia - Revista Eletrônica do Programa de Mestrado em Direitos Humanos, Cidadania e Violência/Ciência Política do Centro Universitário Unieuro

ISSN: 1809-1261

UNIEURO, Brasília, número 27 (Especial), 2019, pp. 188-199.

groups are available, to solve some doubts, questioning and knowledge deal with this carrier in the evolution of the disease.

Keywords: Alzheimer's disease; caregiver; family caregiver; emotional and physical impact; coping with the diagnosis.

\section{INTRODUÇÃO}

A população do mundo e do Brasil estão envelhecendo muito rápido, favorecendo um descontrole para a preparação desse novo ciclo, a mesma proporção em que a população idosa aumenta surgem um crescimento significativo da incidência de doenças crônicas e incapacitantes, inclusive a doença de Alzheimer.

De acordo com o censo demográfico de 2010 (IBGE, 2011), a população brasileira de hoje é de 190.755 .199 milhões de pessoas, sendo que 51\%, o equivalente a 97 milhões, são mulheres e 49\%, o equivalente a 93 milhões, são homens. O quantitativo de pessoas idosas, que, segundo a Política Nacional do Idoso e o Estatuto do Idoso, tem 60 anos ou mais, é de 20.590 .599 milhões, ou seja, aproximadamente $10,8 \%$ da população total. Desses, 55,5 \% (11.434.487) são mulheres e 44,5\% (9.156.112) são homens. (KUCHEMANN, 2011).

Estima-se para 2020, no Brasil, o maior país em extensão territorial e em número de residentes na América Latina, que 29,8\% da população contarão com mais de 60 anos, sendo 40,7 milhões dessas pessoas com 80 anos ou mais de idade. O número de idosos no Brasil e de aproximadamente 35,6 milhões com a expectativa de aumentar para 65, 4 milhões até 2030 e para 115,5 milhões até 2050. (MARINS, HANSEL e SILVA et al, 2016).

Com as mudanças populacionais e o aumento da expectativa de vida, surgem diferentes tipos de doenças, entre elas, as demências estão ganhando espaço, sendo que a doença de Alzheimer é líder no ranking, fazendo parte dos questionamentos e entendimentos dos profissionais de saúde, pesquisadores, sociedade, comunidade e principalmente a família.

A doença de Alzheimer (DA) é um transtorno neurodegenerativo progressivo e fatal, que, se manifesta por deterioração cognitiva e da memória, comprometimento progressivo das atividades de vida diária e uma variedade de sintomas neuropsiquiátricos, além e de alterações comportamentais. (Portaria SAS/ 2013). É uma demência que proporciona ao portador perdas significativas do social, não participação de decisões importantes sobre como será sua vida e a perda da própria identidade.

DA além de causar impacto devastador para o individuo que desenvolve a patologia, resulta em transtorno para a família, sociedade, bem como os serviços públicos e privados, 
Artigo Original

Hegemonia - Revista Eletrônica do Programa de Mestrado em Direitos Humanos, Cidadania e Violência/Ciência Política do Centro Universitário Unieuro

ISSN: 1809-1261

UNIEURO, Brasília, número 27 (Especial), 2019, pp. 188-199.

principalmente nas políticas públicas no sistema único de saúde (SUS). Ainda existem poucas informações para preparar todos que estão envolvidos nesse ciclo, e falta de preparo resulta em onerosos gastos púbicos, além de despesas exacerbadas a família e a pessoa.

As reações familiares frente à notícia da demência apresentam com vários pontos negativos, pois o cuidador familiar não esta preparado para tais mudanças na vida do portador. Uma delas é a inversão de papéis, onde muitas vezes o chefe da família passa a ser completamente dependente do outro necessitando de todo acompanhamento para atividades básicas da vida diária.

A DA acomete uma serie de pessoas no mundo é uma doença com caráter psicossomático muito serio, é debilitante e incapacitante, causa um enorme sofrimento para o portador, mas também causa diversos prejuízos emocionais e físicos aos seus familiares, principalmente ao familiar escolhido para ser o cuidador oficial desse paciente. Deste modo apresentar este assunto frente as dúvidas e anseios do cuidador torna-se imprescindível, para lidar com o portador.

Considerando a necessidade de atualização constante dos profissionais de saúde, familiares e sociedade no atendimento dessa demência, devido uma quantidade insuficiente de artigos para esta temática existe a importância de mais literaturas, pesquisas e evidências que preparam e discutem o assunto com total irrelevância para difundir o conhecimento. Dessa maneira deve-se avançar na busca com trabalhos voltados para orientar e difundir novas descobertas e intervenções significativas, diante dessa realidade, realizou-se uma revisão de literatura. O objetivo central desse trabalho é discutir o impacto da doença de Alzheimer (DA) frente à família e/ ou cuidador. Tendo como objetivos específicos:Descrever a patologia da Doença de Alzheimer (DA); Analisar e apresentar com base na literatura científica o impacto que a doença de DA causa no portador, bem como no cuidador familiar e Abordar o tratamento da DA e as intervenções ao portador, bem como o cuidador familiar.

A respeito da metodologia utilizada para construir um estudo qualificado de informações e embasamento científico foram utilizados trabalhos qualificados que ressaltam a necessidade de trabalhos voltados a esse assunto.

Trata-se de revisão da Literatura onde foram selecionados artigos organizados em bases de dados como Scielo, Lilacs, revistas Periódicas, Teses, Livro de tratado de Geriatria e Gerontologia, Protocolos e Diretrizes do Ministério da Saúde, site virtual da Associação Brasileira do Alzheimer, publicados entres os critérios de seleção foi cadastrado o intervalo de tempo correspondente nos anos de 2010 a 2017. Foram utilizados os seguintes descritores: Doença de 
Artigo Original

Hegemonia - Revista Eletrônica do Programa de Mestrado em Direitos Humanos, Cidadania e Violência/Ciência Política do Centro Universitário Unieuro

ISSN: 1809-1261

UNIEURO, Brasília, número 27 (Especial), 2019, pp. 188-199.

Alzheimer, Cuidador, Cuidador familiar, impacto emocional e Físico, enfrentamento diante do diagnóstico.

Para critérios de Inclusão utilizados foram: artigos originais que respondessem ao objetivo principal, com resumos apresentados na base de dados. E os critérios de exclusão foram: artigos de revisão da literatura e artigos que não respondessem ao objetivo proposta por este estudo.

A Seleção dos artigos para composição do trabalho foi realizada primeiramente pela leitura dos títulos, aqueles que não atenderam ao objetivo da pesquisa foram excluídos. Dos que ficaram, foi lido o resumo de todos os estudos encontrados na busca em cada uma das bases de dados. Foram descartados os artigos cujos resumos não entraram em consonância com a proposta desta revisão, o passo seguinte foi selecionar os artigos na íntegra e analisá-los.

\section{REFERENCIAL TEÓRICO}

2.1 A Doença de Alzheimer e seus problemas

A Doença de Alzheimer acarreta vários agravos para a vida da pessoa portadora e dos familiares. Com o avanço da patologia o indivíduo dependerá mais de intensivos cuidados devido a limitações imposta pela doença.

A fase inicial do DA dura em média, de 2 a 3 anos, apresentando sintomas como comprometimento da memória, principalmente de memória declarativa episódica, surgem dificuldade para recordar datas, compromissos, nomes familiares e fatos recentes, e podem vir acompanhadas da incapacidade de reconhecer a gravidade da problemática da doença. Na fase intermediária, cuja duração varia de 2 a 10 anos, surge uma deterioração mais acentuada dos déficits cognitivos, com sintoma de apraxia, agnosia, alterações visuoespaciais e a depressão está muito presente nesta fase. Por último na fase avançada ou estágio terminal com duração média de 8 a 12 anos, todas as funções cognitivas estão gravemente comprometidas, o portador da doença estará dependentes para todas as atividades básicas da vida diária. (FREITAS, 2016, p. 246 a 247).

Segundo a Associação Brasileira de Alzheimer (ABRAZ, 2018), os estágios e os sintomas mais comuns na fase Inicial raramente são percebidos, como o começo da doença é gradual, é difícil ter certeza exatamente de quando irão apresentar alguns desses sintomas:

Ter problemas com a propriedade da fala (problemas de linguagem);

Ter perda significativa de memória - particularmente das coisas que acabam de acontecer; Não saber à hora ou o dia da semana; 
Artigo Original

Hegemonia - Revista Eletrônica do Programa de Mestrado em Direitos Humanos, Cidadania e Violência/Ciência Política do Centro Universitário Unieuro

ISSN: 1809-1261

UNIEURO, Brasília, número 27 (Especial), 2019, pp. 188-199.

Ficar perdida em locais familiares;

Ter dificuldade na tomada de decisões;

Ficar inativa ou desmotivada;

Apresentar mudança de humor, depressão ou ansiedade;

Reagir com raiva incomum ou agressivamente em determinadas ocasiões;

Apresentar perda de interesse por hobbies e outras atividades

No estágio intermediário como a doença progride, as limitações ficam claras e mais graves, deixando a pessoa mais dependente para as atividades básicas diárias:

Pode ficar muito desmemoriada, especialmente com eventos recentes e nomes das pessoas;

Pode não gerenciar mais viver sozinha, sem problemas;

É incapaz de cozinhar, limpar ou fazer compras.

Pode ficar extremamente dependente de um membro familiar ou do cuidador;

Necessita de ajuda para a higiene pessoal, isto é, lavar-se e vestir-se;

A dificuldade com a fala avança;

Apresenta problemas como perder-se e de ordem de comportamento, tais como repetição de perguntas, gritar, agarrar-se e distúrbios de sono;

Perde-se tanto em casa como fora da casa;

Pode ter alucinações (vendo ou ouvindo coisas que não existem).

Com o estágio avançada seguem totalmente dependentes e apresenta inatividade, o cuidador familiar e o demais profissionais sentem mais dificuldade para lidar com o portador, os sintomas mais evidentes:

Ter dificuldade para comer;

Ficar incapacitada para comunicar-se;

Não reconhecer parentes, amigos e objetos familiares;

Ter dificuldade de entender o que acontece ao seu redor;

É incapaz de encontrar o seu caminho de volta para casa;

Ter dificuldade para caminhar;

Ter dificuldade na deglutição;

Ter incontinência urinária e fecal;

Manifestar comportamento inapropriado em público;

Ficar confinada a uma cadeira de rodas ou cama. 
Artigo Original

Hegemonia - Revista Eletrônica do Programa de Mestrado em Direitos Humanos, Cidadania e Violência/Ciência Política do Centro Universitário Unieuro

ISSN: 1809-1261

UNIEURO, Brasília, número 27 (Especial), 2019, pp. 188-199.

Apresentando todo este quadro desde a fase inicial a fase grave da doença o paciente vai demandar um cuidar diferenciado, devido às limitações geradas pela total dependência de uma assistência.

De acordo com ADACHIA (2013), a Doença de Alzheimer é progressiva, evolui conforme a intensidade do quadro. Desta forma a doença possui os estágios leve, moderado e grave, conforme a progressão dos sintomas. Por ser uma doença degenerativa e incapacitante, atinge não somente a memória, que é comprometida, mas também muitas outras funções vitais são prejudicadas.O aspecto mais grave é que a doença é irreversível, não existindo até o momento a cura. Os tratamentos utilizados atualmente contribuem para que os sintomas sejam amenizados, e a doença evolua mais lentamente.

\subsection{A Doença de Alzheimer frente ao Cuidador familiar}

A DA pode causar na família do portador inúmeros questionamentos e desestrutura das pessoas envolvidas em relação a situações a qual serão apresentadas, como a falta de conhecimento da gravidade da patologia, ao enfretamento emocional do cuidador e o paciente, e a troca de inversão de papéis, levando o cuidador a depara-se com uma fragilidade física, mental e emocional.

A partir do momento que o idoso é diagnosticado com a doença e a possível necessidade para todos os cuidados, podem surgir conflitos familiares na definição do cuidador, um possível estresse na pessoa que assumirá este papel devido a sua falta de conhecimento para a função.

Geralmente a pessoa que assume o papel de cuidador possui idade avançada, além de toda responsabilidade, sobrecarga e solidão implícitas na função, deve conviver com o próprio envelhecimento, limitações e responsabilidades financeiras. (MAZOCO et al., 2017, p. 70).

O cuidar de um familiar com Doença de Alzheimer exige que o cuidador aprenda a conviver com o sofrimento e com as dependências do portador, e por muitas vezes esconde a sua própria dor e suas necessidades, pois é preciso fazer quase tudo para o outro, mesmo conhecendo quase nada do que será vivenciado. (KUCMANSKI e colaboradores, 2016, p.6).

A família que atende ao doente, sofre desequilíbrios ou alterações comportamentais em todos os membros que a integram, levando muitas vezes uma separação ou total ruptura da família, gerando uma problemática psicopatológica muito mais ampla do esperado. Através desse descontrole afetará possivelmente o portador, acelerando um processo neurodegenerativo. (RIBEIRO, 2010, p. 17). 
Artigo Original

Hegemonia - Revista Eletrônica do Programa de Mestrado em Direitos Humanos, Cidadania e Violência/Ciência Política do Centro Universitário Unieuro

ISSN: 1809-1261

UNIEURO, Brasília, número 27 (Especial), 2019, pp. 188-199.

De acordo com Ximenes e colaboradores (2014), as exigências dos cuidados pela doença e seu abalo emocional na vida do cuidador familiar, dá uma noção da proporção das dificuldades que serão vivenciadas no dia a dia pelas famílias. Diante deste cenário segundo Kucmanski e colaboradores(2016), surge o cuidador como peça principal nos estágios leve, moderado e avançado, sendo o estágio final o mais doloroso. Através desse comprometimento do indivíduo nomeiam um o responsável para auxiliar, realiza e direcionar os cuidados básicos de vida diária.

Para (Anadão; Vitor, 2014, p. 113) a DA trazpara os membros de sua família uma série de impactos psicológicos entre os quais sepode citar: os conflitos familiares em virtude do constante cuidado com o paciente,níveis altos de estresse, depressão, os sentimentos de ansiedade, estarrecimento, impotênciae tristeza, os quais tendem a se intensificar à medida que o quadro clínicodo paciente vai se agravando.

A partir dos cuidados com o doente eaos obstáculos enfrentados pela doença o cuidador familiar apresentar varias alterações psicológicas devido a assistência ininterrupta, conflitos com os demais membros da família, a falta de informação, a falta de condições financeiras, falta de lazer e sem vida pessoal, o mesmo sofre impactos negativos como: ansiedade, depressão, estresse e tendo prejuízo em sua qualidade de vida.

\subsection{A Doença de Alzheimer e seu tratamento}

O tratamento da DA envolve estratégias farmacológicas e intervenções psicossociais para o portador e seus familiares, trata-se de uma busca para atenuar a evolução dos sintomas, retardando um pouco o progresso evolutivo da doença.

No campo do tratamento farmacológico, inúmeras substâncias psicoativas têm sido propostas para restabelecer e preservar a cognição, o comportamento e as habilidades básicas do portador do Alzheimer. Já nas intervenções existentes tem muitos praticas sendo desenvolvidas para melhorar a qualidade de vida do portador, bem como preparar o cuidador para impacto emocional que será vivenciado.

De acordo com a Portaria aprovada conjunta $n^{\circ}$ 13, de 28 de novembro de 2017 (MINISTÉRIO DA SAÚDE, 2017), estabelece o novo protocolo clínico e diretrizes terapêuticas para o tratamento da DA. Alguns medicamentos fazem parte para critério de inclusão no tratamento das fases leve, moderada e grave,os mais utilizados na atualidade são os inibidores da acetilcolinesterase donepezila, galantamina, rivastigmina e também a memantina. 
Artigo Original

Hegemonia - Revista Eletrônica do Programa de Mestrado em Direitos Humanos, Cidadania e Violência/Ciência Política do Centro Universitário Unieuro

ISSN: 1809-1261

UNIEURO, Brasília, número 27 (Especial), 2019, pp. 188-199.

Os inibidores da acetilcolinesterase, donepezila, galantamina e rivastigmina, são recomendados para o tratamento da DA leve a moderada. $O$ efeito esperado para o uso de fármacos colinérgicos recai no aumento da secreção ou no prolongamento da meia-vida da acetilcolina na fenda sináptica em áreas relevantes do cérebro. (MS, 2017, p. 10).

Existem ainda em fase de estudos outros fármacos que agem juntamente com medicamentos convencionais entre eles: O Ginkgo Biloba que age na melhora da memória, Vitamina Eo benefício do tratamento com o uso de dose elevada (2000 UI/dia) auxilia no comprometimento cognitivo leve, Ômega 3 atua na prevenção de comprometimento cognitivo ou demência. Estes fármacos necessitam de mais evidências para demonstrar eficácia no tratamento (VALE et al., 2011, p.38).

O tratamento da DA deve ser multidisciplinar, contemplando os diversos sinais e sintomas da doença e suas peculiaridades de condutas. A Finalidade do tratamento farmacológico é atenuar a evolução do comprometimento cognitivo, o ponto de vista do comportamento é manter as habilidades funcionais preservadas, com o mínimo de agravos para o portador. (MS, 2017, p. 10).

Todo o tratamento farmacológico será acompanhado pelos profissionais da área médica que atenderá os sintomas e a progressão da doença, sendo de suma importância o trabalho da equipe multidisciplinar para acompanhar quadro evolutivo do portador.

Além do tratamento de fármacos e substâncias, são disponibilizadas algumas intervenções ao portador sendo a estimulação cognitiva uma das atividades mais importante, pois realiza um treinamento diário, utilizando técnicas com uso de objetos e instrumentos, o objetivo da atividade é a de manter as funções existentes e permitir que elas compensem as funções comprometidas (CRUZ, 2011, p.36-37).

Algumas intervenções também são disponibilizadas para a cuidadores familiares, pois necessitam de acompanhamento para entenderam as complicações e o saber lidar com as dificuldades que o portador irá vivenciar então algumas atividades são oferecidas pela equipe multidisciplinar, para Instituto de psiquiatria do hospital das clínicas da faculdade de medicina da Universidade de São Paulo, são desenvolvidos encontros com os familiares para compreender a patologia, diálogos com relatos e reflexões, trocas de experiências no saber lidar com os cuidados diários as problemáticas enfrentadas (LOPES; CACHIONI, 2013, p. 446).

Para Tristão e Santos (2015, p. 1176), apresenta em sua literatura um grupo de estudo sobre cuidados em saúde de pessoas idosas (GESPI) que acontecem no Hospital universitário de Santa Catarina, onde são realizadas reuniões com a coordenadora enfermeira e a equipe 
Artigo Original

Hegemonia - Revista Eletrônica do Programa de Mestrado em Direitos Humanos, Cidadania e Violência/Ciência Política do Centro Universitário Unieuro

ISSN: 1809-1261

UNIEURO, Brasília, número 27 (Especial), 2019, pp. 188-199.

multidisciplinar acompanhada e vinculada da ABRAZ desde 2004, nesses encontros são oferecidos grupo de apoio de familiares, discutem em relação à patologia, declínio cognitivo, cuidados integrais e emocionais do portador e do familiar.

Estas intervenções oferecidas para os cuidadores familiares tornam-se de suma importância para adequação da família, deixando-os mais seguros e capazes para os cuidados essenciais ao doente.

\section{CONSIDERAÇÕES FINAIS}

Por meio deste estudo pode-se inferir que está aumentando a quantidade de idosos com DA no mundo. É um a doença na qual o portador fica muitas vezes incapacitado e dependente para as necessidades básicas. Os tratamentos farmacológicos têm resultados significativos para atenuar os sintomas e as perdas das habilidades funcionais, bem como as intervenções não farmacológicas que apresentam evidencias comprovadas de melhora da qualidade de vida.

$\mathrm{Na}$ elaboração de deste trabalho evidenciou que a doença de Alzheimer vem crescendo de uma forma rápida e devastadora proporcionando ao doente uma serie de enfrentamentos e dependência para os cuidados básicos e complexos, deixando o mesmo impossibilitado de gerir sua própria vida.Muitas vezes com sérios comprometimentos cognitivos e comportamentais, com isso um cuidador familiar é direcionado para assumir este papel, que será de suma importância para o tratamento e convivência com o portador.

Entretanto o cuidador familiar e toda famílianão estão preparados para esta nova vida, deparam-se com situações de dor e sofrimento, pois em nenhum momentoforam habilitados inicialmente para enfrentar esta realidade. Então quem assiste o portador no decorrer da doença, sofrem muito os impactos emocionais e físicos, por muitas vezes não estão preparados para os efeitos neurodegenerativos progressivos da doença, necessitando de esclarecimento e entendimento.

No entanto, existem algunsgrupos de apoio que são disponibilizados pelas unidades de saúde e instituições os quais realizam o tratamento para o portador da DA, ajudam os familiares a teremum suporte maior para acompanhar a evolução DA. Dessa forma contribui para sanar alguns questionamentos e dúvidaspara todas as fases do declínio da patologia. 
Artigo Original

Hegemonia - Revista Eletrônica do Programa de Mestrado em Direitos Humanos, Cidadania e

Violência/Ciência Política do Centro Universitário Unieuro

ISSN: 1809-1261

UNIEURO, Brasília, número 27 (Especial), 2019, pp. 188-199.

\section{REFERÊNCIAS}

ASSOCIAÇÃO BRASILEIRA DE ALZHEIMER. O que é Alzheimer: Evolução da Doença

Disponível em:http://www.abraz.org.br/.

BRASIL. MINISTÉRIO DA SAÚDE SECRETARIA DE ATENÇÃO A SÁUDE. PORTARIA CONJUNTA N ${ }^{\text {13 }}$, DE 28 DE NOVEMBRO DE 2017.Protocolo Clínico e Diretrizes Terapêuticas da Doença de Alzheimer.

BRASIL. MINISTÉRIO DA SAÚDE SECRETARIA DE ATENÇÃO A SÁUDE. PORTARIA CONJUNTA N 13, DE 21 DE NOVEMBRO DE 2013.Protocolo Clínico e Diretrizes Terapêuticas : Doença de Alzheimer.

CARVALHO DO VALE, Francisco de Assis et al. Tratamento da doença de Alzheimer. Dementia \& Neuropsychologia, v. 5, n. 1, 2011.

CRUZ, Thiara Joanna Peçanha da et al. Avaliação da estimulação cognitiva para o idoso com demência de Alzheimer realizada pelo cuidador no domićlio: uma tecnologia de cuidado em enfermagem. 2011.

DE OLIVEIRA LOPES, Lais; CACHIONI, Meire. Cuidadores familiares de idosos com doença de Alzheimer em uma intervenção psicoeducacional. Revista Brasileira de Geriatria e Gerontologia, v. 16, n. 3, p. 443-460, 2013.

Freitas, Elizabete Viana de. Tratado de Geriatria e gerontologia/ Elizabete Viana de Freitas, Ligia Py, - 4 ed- Rio de Janeiro: Guanabara Koogan, 2016

KÜCHEMANN, Berlindes Astrid. Envelhecimento populacional, cuidado e cidadania: velhos dilemas e novos desafios. Sociedade e Estado, v. 27, n. 1, p. 165-180, 2012.

MAZOCO, V. A., SUGUIHURA, A. L. M. \& WECHSLER, A. M. “

Impacto psicológico em cuidadores de pessoas com a doença de Alzheimer, in Psicologia Saberes \& Práticas, $n .1$, v.1. 2017. p. 69-76

RIBEIRO, CLÉRIS FERREIRA. Doença de Alzheimer: Aprincipal causa de demencia nos idosos e seus impactos na vida familiares e cuidadores. 2010.

REIS TRISTÃO, Francisco; AZEVEDO DOS SANTOS, Silvia Maria. Atenção ao familiar cuidador de idoso com doença de alzheimer: Uma atividade de extensão universitária. Texto \& Contexto Enfermagem, v. 24, n. 4, 2015.

SALETE KUCMANSKI, Luciane et al. Doença de Alzheimer: desafios enfrentados pelo cuidador no cotidiano familiar. Revista Brasileira de Geriatria e Gerontologia, v. 19, n. 6, 2016. 
Artigo Original

Hegemonia - Revista Eletrônica do Programa de Mestrado em Direitos Humanos, Cidadania e Violência/Ciência Política do Centro Universitário Unieuro

ISSN: 1809-1261

UNIEURO, Brasília, número 27 (Especial), 2019, pp. 188-199.

VITOR, Thayná Lima Pereira; ANADÃO, Nara Rocha. Familiares de idosos com a doença de Alzheimer: uma reflexão sobre aspectos psicossociais. Caderno de Graduação-Ciências Biológicas e da Saúde-UNIT-ALAGOAS, v. 2, n. 2, p. 111-130, 2014.

XIMENES, Maria Amelia; RICO, Bianca Lourdes Duarte; PEDREIRA, Raíza Quaresma. Doença de Alzheimer: a dependência e o cuidado. Revista Kairós: Gerontologia, v. 17, n. 2, p. 121-140, 2014. 